\title{
1 Elucidating the Electronic Structure of Supported Gold Nanoparticles 2 and its Relevance to Catalysis by Means of Hard X-ray Photoelectron

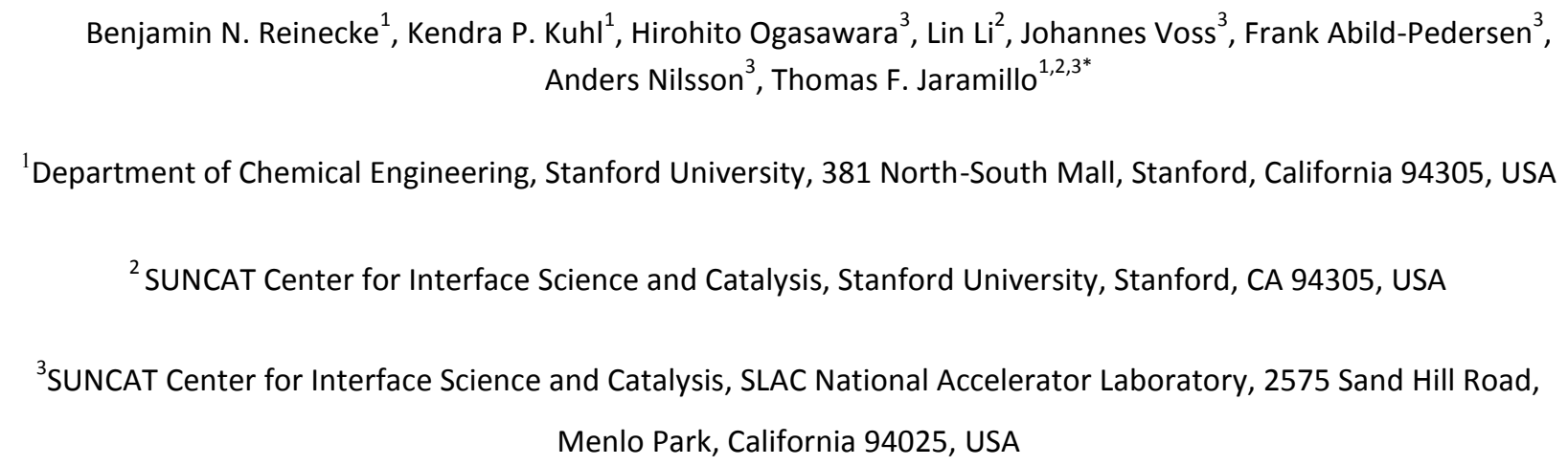

We report on the electronic structure of $\mathrm{Au}$ (gold) nanoparticles supported onto $\mathrm{TiO}_{2}$ with a goal of elucidating the most important effects that contribute to their high catalytic activity. We synthesize and characterize with high resolution transmission electron microscopy (HRTEM) 3.4, 5.3, and $9.5 \mathrm{~nm}$ diameter $\mathrm{TiO}_{2}$-supported Au nanoparticles with nearly spherical shape and measure their valence band using Au 5d subshell sensitive hard X-ray photoelectron spectroscopy (HAXPES) conducted at Spring-8. Based on Density Functional Theory (DFT) calculations of various Au surface structures, we interpret the observed changes in the Au $5 \mathrm{~d}$ valence band structure as a function of size in terms of an increasing percentage of $\mathrm{Au}$ atoms at corners/edges for decreasing particle size. This work elucidates how $\mathrm{Au}$ coordination number impacts the electronic structure of Au nanoparticles, ultimately giving rise to their well-known catalytic activity.

(1)

Gold Nanoparticles, Transmission Electron Microscopy, Valence Band, Support effect, Titanium Dioxide, Hard X-ray Photoelectron Spectroscopy.

\section{$\underline{\text { Key words }}$}


At this point in time it has been well-established that whereas bulk gold is catalytically inert, gold nanoparticles $<10 \mathrm{~nm}$ diameter supported onto metal oxides can be highly catalytically active for a number of reactions, including low temperature CO oxidation[1], Water Gas Shift (WGS)[2], and NO

\section{Geometric Effects}

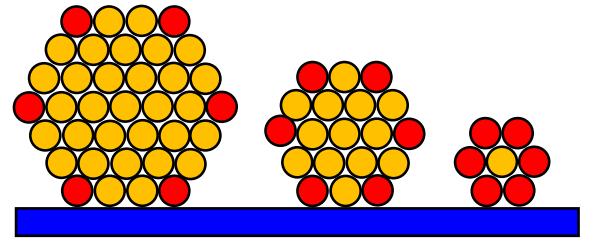

1a. \# Undercoordinated Sites: Size 1b. \# Undercoordinated Sites: Shape

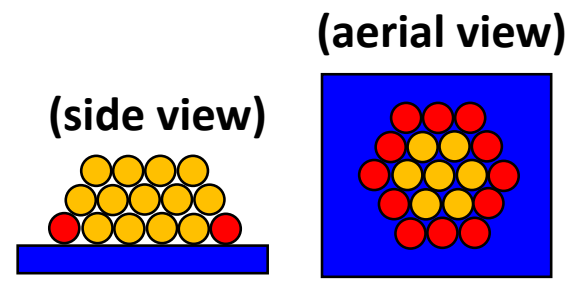

3. Perimeter sites

\section{Electronic Effects}

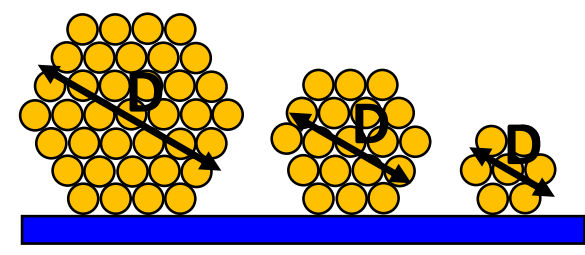

5. Electronic quantum size 
Figure 1. Nanoscaling Origins of Au Catalysis.

Schematic showing possible nano-scale origins of gold catalysis. Red atoms in $1 \mathrm{a}$ and $1 \mathrm{~b}$ indicate undercoordinated atoms. Red atoms in 3 indicate perimeter sites.

\begin{tabular}{|c|c|c|}
\hline \multicolumn{2}{|l|}{ Geometric Effects } & Associated References \\
\hline \multirow{2}{*}{$\begin{array}{l}\text { 1. Exposing catalytically } \\
\text { active/undercoordinated gold sites by changing: }\end{array}$} & a. Size & {$[7,8]$} \\
\hline & \begin{tabular}{|l|} 
b. Shape \\
\end{tabular} & {$[8-11]$} \\
\hline \multicolumn{2}{|l|}{ 2. Lattice strain } & {$[11,12]$} \\
\hline \multicolumn{2}{|l|}{ 3. Unique gold/support perimeter sites } & [7] \\
\hline \multicolumn{2}{|l|}{ Electronic Effects } & \\
\hline \multicolumn{2}{|l|}{ 4. Charge state } & [13-18] \\
\hline \multicolumn{2}{|l|}{ 5. Quantum size } & [19-22] \\
\hline
\end{tabular}

Table 1. Leading nanoscaling effects in gold catalysis.

The nanoscaling effects are broken down into geometric and electronic effects, each with selected references from the literature that support the importance of the effect.

In short, there are two general classifications of nano-scale effects, those that are predominantly geometric in nature and those that are electronic in nature. The predominantly geometric effects have to do with the physical orientation of Au atoms that can change with size[7, 8], shape[8-11], lattice strain[11, 12], and the Au-support perimeter[7]; while the predominantly electronic effects can arise from charge transfer to/from the support[13-18], or from quantum size effects[19-22].

With so many proposed explanations for the high activity of small supported gold nanoparticles, an approach is needed to experimentally disentangle these effects and assess which of these are dominant in contributing to catalytic activity; not a trivial undertaking as many of the effects are correlated with one another. 
Critical to sorting out these effects is understanding the valence band electronic structure of

58 supported Au nanoparticles. This, along with detailed knowledge of the physical structure of the nanoparticles regarding size and shape, can be particularly helpful in understanding trends in catalysis[23-26]. This approach has been applied in the field of catalysis over a wide range chemical and

61 electrochemical reactions, e.g. the oxygen reduction reaction (ORR) in fuel cells[27] and CO oxidation in conventional heterogeneous catalysis[24].

To this end, a number of studies have measured the valence band electronic structure of Au nanoparticles supported onto $\mathrm{TiO}_{2}[19,28], \mathrm{Al}_{2} \mathrm{O}_{3}[29]$, and $\mathrm{NiO}[16]$ using Ultraviolet Photoelectron Spectroscopy (UPS, 40 eV) and Au nanoparticles supported onto carbon [30-33] using conventional Al K- $\alpha$ X-ray Photoelectron Spectroscopy (XPS, $1486 \mathrm{eV).} \mathrm{UPS} \mathrm{and} \mathrm{conventional} \mathrm{XPS} \mathrm{have} \mathrm{been} \mathrm{valuable} \mathrm{in}$ understanding the electronic structure of Au nanoparticles. One key point resulting from these studies is that a metal-insulator transition occurs as a function of size, observed between $\mathrm{Au}_{55}$ and $\mathrm{Au}_{33}$, ascribed to quantum size effects. However, UPS and conventional XPS have their limitations, as it has been found that for many supports the main spectral features of the Au $d$ band are generally difficult to distinguish

71 as the nanoparticle size decreases or as gold coverage decreases, due to a heavy background valence 72 band signal from the support that overlaps with the signal from the Au nanoparticles[16, 19, 28-30] .

Another means to study $\mathrm{Au} d$ band structure is through electronic structure theory calculations. Density functional theory (DFT) calculations have revealed that undercoordinated gold atoms have $d$ states that are higher in energy than their corresponding full coordinated bulk gold atoms [10] and thus bind adsorbates such as $\mathrm{O}_{2}$ more strongly. This provides a compelling explanation for the enhanced $\mathrm{CO}$ 77 oxidation activity of small gold nanoparticles.

Given the importance of a catalyst's electronic structure in determining its catalytic activity, there 
the metal oxide support signal, and with high-energy resolution. We accomplish this by using an

81 unconventional form of XPS, the hard X-ray (8 KeV) photoelectron spectroscopy (XPS) beam-line (BL47)

82 at the Spring8 Synchrotron. Due to the proportionately large photoionizaiton cross-section of the Au 5d

83 subshell versus those of the metal oxide support at this photon energy, we are able to straightforwardly

84 deconvolute any signal from the metal oxide support in the valence band that could otherwise

85 overwhelm the valence band signal from a submonolayer of Au nanoparticles. Our study also employs

86 DFT calculations to help interpret the spectral features that we observe. For our studies, we chose $\mathrm{TiO}_{2}$

87 as a support since it is known as one of the more active supports for Au nanoparticle catalysis and does

88 not contribute to CO oxidation on its own[34]. In this manuscript, we aim to fill the gaps in our current

89 knowledge within the area of Au nanoparticle catalysis by addressing the following two key questions

90 for Au nanoparticles supported onto $\mathrm{TiO}_{2}$ : (1) How does the physical structure of the Au nanoparticles,

91 e.g. size, shape, and lattice constant/strain, influence its valence band electronic structure? (2) How can

92 knowledge of the electronic structure help sort out the different possible origins of catalytic activity, and

93 help determine which are most important within our system of study?

Our approach to answering these questions is outlined here. We started by synthesizing sizecontrolled Au nanoparticles onto a flat, well-defined, conductive $\mathrm{TiO}_{2}$ support by means of a ligand-free synthetic method in an effort to avoid organic contaminants. High-Resolution Transmission Electron

97 Microscopy (HRTEM) was used to measure the size, shape, and lattice constant/strain of the supported 98 gold nanoparticles. With detailed knowledge of these physical characteristics, we then measured the 99 electronic structure of the $\mathrm{Au}$ nanoparticles by means of hard X-ray ( 8 KeV) photoelectron spectroscopy (XPS). With this incident photon energy, the ratio of the absorption cross-section for the

101 valence $5 d$ electrons of gold to the photoionizaiton cross-section for the $02 p$ and Ti $3 d$ valence 102 electrons from the $\mathrm{TiO}_{2}$ support is predicted (figure 2) to be an order of magnitude greater than with 
$104 \mathrm{eV})[35]$.

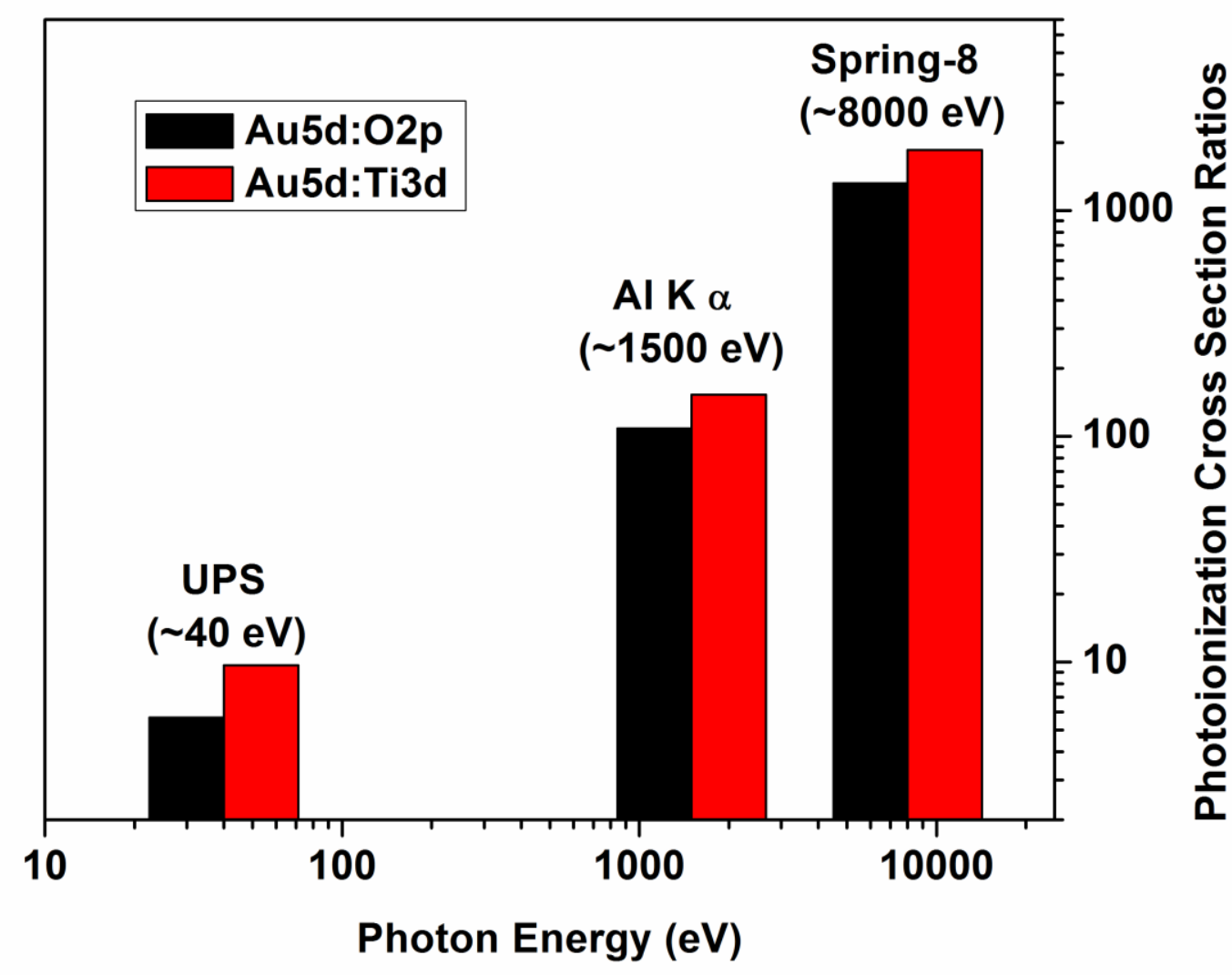

Figure 2. Photoionization Cross Section Ratios.

Theoretical calculation of the Au $5 d / O 2 p$ and $A u 5 d / T i$ 3d photoelectron cross section ratios at three different photon energies ( $40 \mathrm{eV}, \sim 1500 \mathrm{KeV}$, and $28000 \mathrm{eV})[35]$.

110 Using hard X-rays allowed us to see an enhanced signal from the gold versus the background $\mathrm{TiO}_{2}$, which

111 to the best of our knowledge has not yet been reported for catalytically relevant oxide supported gold

112 nanoparticles. Based on our findings described below, we believe that selectively probing $5 \mathrm{~d}$ transition 
metal catalysts on oxide supports could become very important to achieve the objective of correlating valence band structure to catalytic performance in many areas of catalysis.

\section{Experimental}

\subsection{Sample Preparation}

First, $1400 \AA$ of titanium was deposited by electron beam evaporation onto silicon(111) $(0.001 \mathrm{ohm} * \mathrm{~cm})$ in a clean room environment using an Innotec ES26C evaporation system. The rate of deposition was $0.15 \AA /$ second for 5 minutes and then ramped to $2 \AA /$ second for the remainder of the deposition. The chamber temperature and pressure were $45^{\circ} \mathrm{C}$ and $2 \times 10^{-7}$ torr, respectively. The titanium film was then exposed to ambient air to form a thin $\mathrm{TiO}_{2}$ native oxide. Finally, 3, 8, and $20 \AA$ nominal thicknesses of gold were electron beam deposited at a rate of $0.15 \AA$ /second onto several titanium-metalized Si wafers to achieve three different sized gold nanoparticles on $\mathrm{TiO}_{2}$, respectively. The chamber temperature and pressure were $30^{\circ} \mathrm{C}$ and $2 \times 10^{-7}$ torr during Au deposition. The remaining gold-free, titanium metalized $\mathrm{Si}$ wafers provided background controls for electronic structure measurements. The bulk gold surface studied in this investigation consisted of an evaporated film of gold on a copper substrate that is stored inside the ultra-high vacuum chamber at beam-line $47 \mathrm{XU}$ at the Spring8 Synchrotron. That sample will be referred to as "bulk gold" from this point on.

\subsection{Transmission Electron Microscopy (TEM)}

The supported gold nanoparticles were examined in an FEI Tecnai G2 F20 X-Twin Transmission Electron Microscope (TEM) to determine the size distribution and shape. Both plan view (top-down) and cross sectional view (side-on) samples are prepared using standard TEM preparations which included: heating 
134 of the sample with crystal bond to $180^{\circ} \mathrm{C}$ for use as a binder, SiC film lapping, diamond film lapping, 135 alumina slurry dimpling, and Ga ion milling.

\subsection{Electronic structure measurements}

137 The valence band and the Au $4 \mathrm{f} \mathrm{X}$-ray photoelectron spectra for the $\mathrm{TiO}_{2}$ bare support, the three 138 different $\mathrm{TiO}_{2}$ supported gold nanoparticle samples, and bulk gold were measured at the Spring-8 139 Synchrotron, BeamlineBL47XU, the hard x-ray photoelectron spectroscopy (HAXPES) beam-line, at a 140 photon energy of $7945 \mathrm{eV}(\sim 8 \mathrm{KeV})$. Photoelectrons were collected using a GammadataScienta R4000 141 spectrometer with a total resolution better than $0.25 \mathrm{eV}$. Each photoelectron spectrum was measured 142 by illuminating the $\mathrm{x}$-ray beam at $1^{\circ}$ incidence to the surface and collecting the appropriate 143 photoelectrons at $89^{\circ}$ to the surface plane. Additionally, the Ti $2 p$ region was collected for analysis of 144 the $\mathrm{TiO}_{2}$ support and to rule out any charging induced binding energy shifts or broadening among the 145 XPS spectra of the gold nanoparticles.

\subsection{Electronic structure calculations}

147 The electronic structures of Au surfaces with various coordination numbers were determined from 148 density functional theory (DFT) calculations using the Quantum Espresso code[36]. The (100), (111), and 149 (211) surfaces were modeled using $2 \times 2 \times 6$ slabs, with $20 \AA$ of vacuum. The (421) surface was modeled 150 using a 36 atom $(1 \times 1)$ surface unit cell, containing five close-packed layers, with $13 \AA$ of vacuum. A 151 Crystallographic Information File (CIF) of each model is provided as Supporting Information: Au100.cif, 152 Au111.cif, Au211.cif, Au421.cif, and Aubulk.cif. For all surfaces, Brillouin zone sampling with grid153 spacings of no more than $0.051 / \AA ̊$ with Fermi smearing of $\mathrm{kBT}=0.1 \mathrm{eV}$ was used, and the Kohn-Sham 154 bands were expanded in plane wave basis sets with $500 \mathrm{eV}$ cut off. Static calculations were carried out 155 using the experimental lattice constant, $4.08 \AA[34]$, and for $3 \%$ compressive strain (in all X-Y-Z). 156 Generalized Gradient Approximation (PBE $[35,36]$ ) was used to describe the exchange-correlation 
effects. Spin-orbit coupling was taken into account by using an ultrasoft pseudopotential with $j$ -

158 dependent projectors [37, 38]

\section{Results \& Discussion}

Electron beam evaporation produces size-controlled gold nanoparticles with a very high surface density, as depicted in the TEM images in Figure 3. Both low magnification and high-resolution (HRTEM) images of the three samples in plan view are shown in Figure $3(1-\mathrm{A}, 1-\mathrm{C}$, and 1-E) and Figure 3 (2average diameters: $3.4 \mathrm{~nm}, 5.3 \mathrm{~nm}$, and $9.5 \mathrm{~nm}$ with an error in the mean of $0.1 \mathrm{~nm}, 0.1 \mathrm{~nm}$, and $0.2 \mathrm{~nm}$, respectively. These results agree well with SEM observations (see Supporting Information). support and the shape of the gold nanoparticles, shown in Figure 3 (1-B, 1-D, and 1-F and 2-B, 2-D, and 2-F). The native $\mathrm{TiO}_{2}$ layer (see Supporting Information) can be seen clearly by the contrast between the 171 polycrystalline titanium phase and the $\mathrm{TiO}_{2}$ layer on top from Figure $3(1-\mathrm{F})$. The thickness of the $\mathrm{TiO}_{2}$ 172 layer is roughly $6 \mathrm{~nm}$, indicated by a pair of arrows. The thickness as measured by XPS (see Supporting 173 Information) is $1.1 \mathrm{~nm}$. The difference between the XPS and TEM derived thickness measurements is 174 most likely due to the $180^{\circ} \mathrm{C}$ heating step during TEM preparation, which likely causes growth in the $\mathrm{TiO}_{2}$ 175 layer. The cross sectional TEM images show that the gold nanoparticles bind to the $\mathrm{TiO}_{2}$ surface in a 176 spherical but slightly flattened form (see Supporting Information). We have ruled out significant shape 177 change or sintering of the gold nanoparticles during TEM preparation or imaging based on comparisons 178 of size and surface density (\# particles/area) in SEM versus TEM (see Supporting Information). 

are used throughout the discussion. Histograms of each nanoparticle size and associated standard deviation as determined by TEM plan view imaging is shown in the insets of figure 3 (1-A, 1-C, and 1-E).

182 It has been shown previously that the truncated octahedron geometry fits well for small nanoparticles of 183 face centered cubic (FCC) metals such as gold[39] and has been used to model supported gold

\section{Model: Truncated} Octahedron
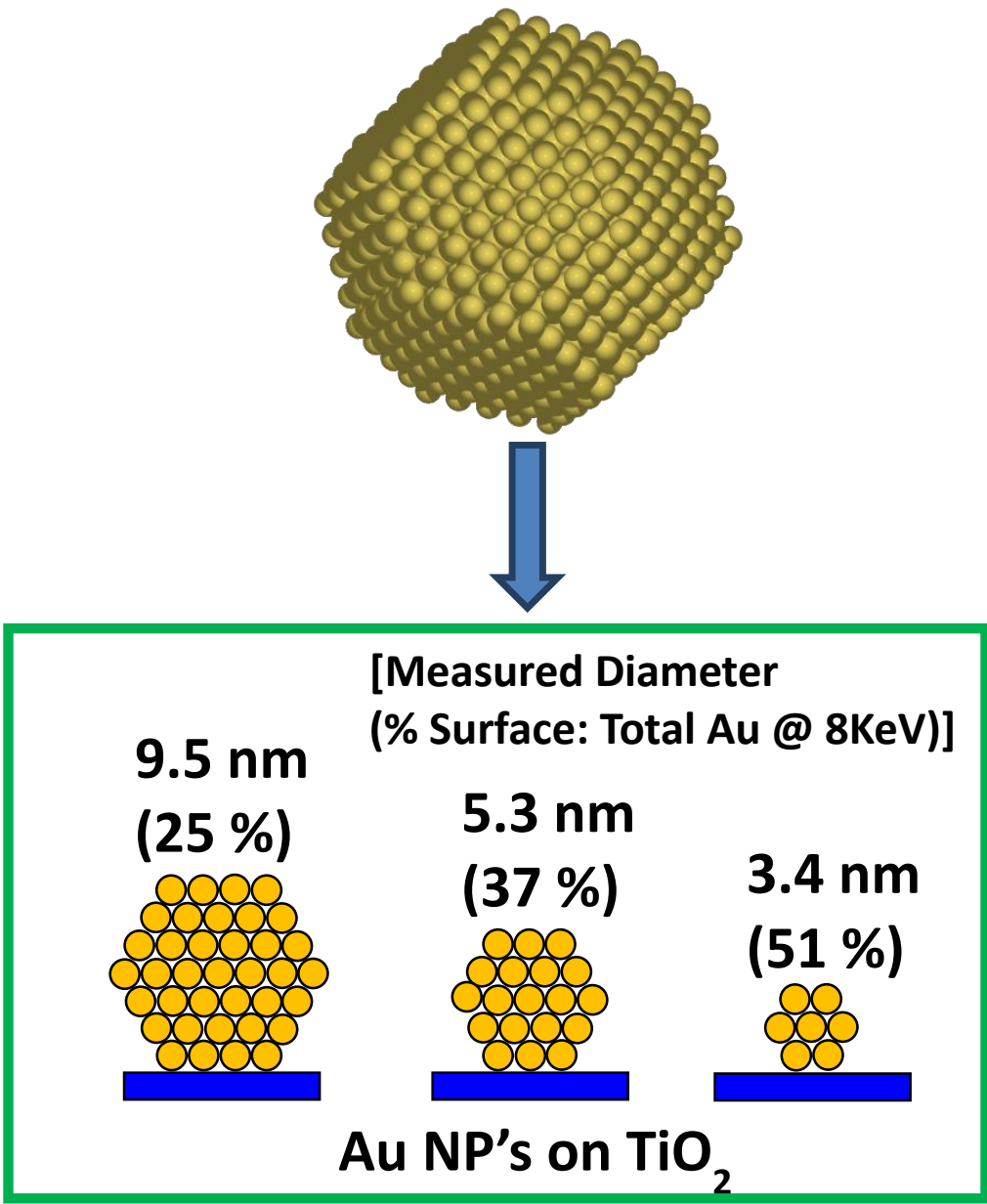

Figure 4.Truncated Octahedron Model. 

nanoparticles of each size (3.4 nm, $5.3 \mathrm{~nm}, 9.5 \mathrm{~nm}$ diameter).

190 Shape change with size due to the support (nanoscaling effect \#1b, Table 1) is therefore not an 191 important consideration for these gold nanoparticles.

\subsection{Electronic Structure Characterization}

\subsubsection{Au 4f XPS}

194 Core-level shifts in the Au $4 \mathrm{f}$ spectral region have previously been used to describe changes in chemical 195 state [40-43] or geometric arrangement $[41,44,45]$ of the Au surface. For all of our samples, including 196 bulk gold and the three $\mathrm{TiO}_{2}$-supported gold nanoparticle samples, the $\mathrm{Au} 4 \mathrm{f}$ core level measurements 197 from figure 5 show only one doublet with the $A u 4 f_{7 / 2}$ binding energy at $84.0 \pm 0.1 \mathrm{eV}$. 


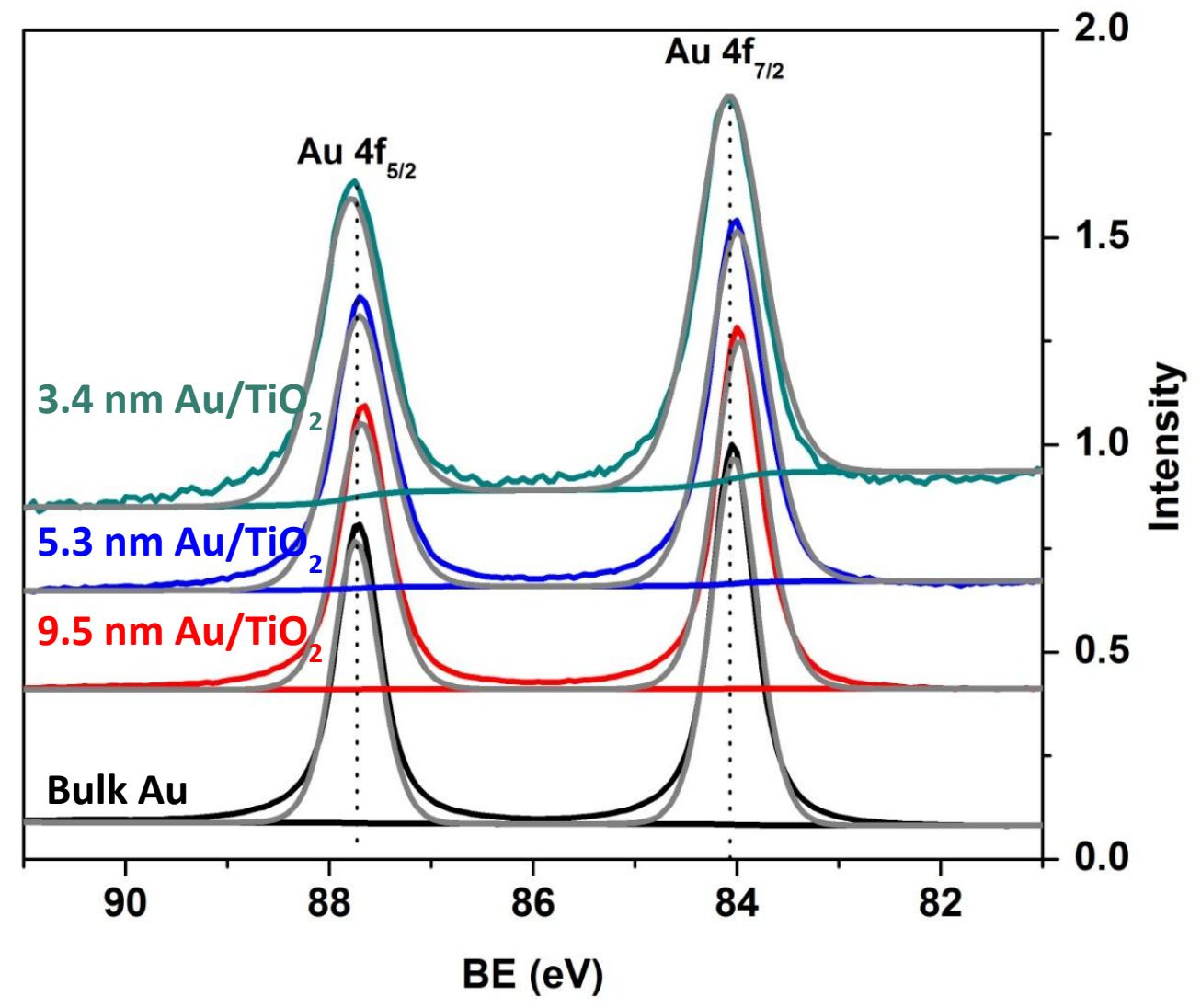

Figure 5. Au 4f XPS.

Au $4 f$ core level spectra collected using 8 KeV hard X-raysfor each of the $3.4 \mathrm{~nm}, 5.3 \mathrm{~nm}$, and $9.5 \mathrm{~nm}$ average diameter $\mathrm{TiO}_{2}$-supported gold nanoparticles as well as bulk gold. Intensities have been normalized.

This agrees well with measurements on bulk metallic gold, with an $A u 4 f_{7 / 2}$ binding energy also at 84.0 eV[46], supporting the notion that all the Au nanoparticle samples are in a charge-neutral, bulk-like state. Metallic (charge-neutral) gold has been shown to be the catalytically active state for CO oxidation [47]. It should be noted that some supported gold nanoparticles in this size regime, synthesized by other methods, have been reported as having a shell of oxidation [40]. In our case there is no observable oxidation at the surface, as this would manifest itself as additional $A u 4 f_{7 / 2}$ and $A u 4 f_{5 / 2}$ 
contributions upshifted significantly in binding energy. Others have shown that there are a number of

211 possible final and initial state effects that can shift the binding energy either up or down [41]. Final state

212 (screening) effects are expected to be minute at this size regime [48], especially on a conductive 213 support[41].

From the initial (electronic) state viewpoint, it has been found that oxygen defect sites on the

$215 \mathrm{TiO}_{2}$ support can anchor nanoparticles to the surface while polarizing the gold atoms bound to those 216 anchor sites. Given the size and shape of the nanoparticles in the present study, however, the fraction

217 of gold atoms affected in this manner is expected to be miniscule [15] and any binding energy shift due 218 to these atoms would likely be outside of the detection capability of XPS. For example, only 3-4 atoms 219 of Au would be affected for a $3 \mathrm{~nm}$ spherical gold nanoparticle on $\mathrm{TiO}_{2}$ [15]. Given the size range studied 220 here $(3.4 \mathrm{~nm}, 5.3 \mathrm{~nm}$, and $9.5 \mathrm{~nm})$, electronic promotion effects are expected to be negligible as such 221 effects have previously been found to be insignificant for gold nanoparticles above 2-3 nm in diameter 222 on a number of oxide supports [17]. While a surface core level shift of $0.4 \mathrm{eV}$ has been observed before 223 for a gold surface [44], in our case we do not observe such an effect. To explore whether increased core 224 level line broadening due to the heterogeneity of surface sites for a given nanoparticle size and the finite 225 size distribution of nanoparticles could potentially mask such subtle shifts[41], we carefully examined 226 line broadening in our samples. We observed line broadening for the smaller Au nanoparticles by virtue 227 of an increasing full width at half $\max (\mathrm{FWHM})$ with decreasing nanoparticle size: The FWHM of the Au $2284 \mathrm{f}_{7 / 2}$ peak was measured as $0.54 \mathrm{eV}, 0.60 \mathrm{eV}, 0.68 \mathrm{eV}$, and $0.77 \mathrm{eV}$ for bulk gold, $9.5 \mathrm{~nm}, 5.3 \mathrm{~nm}$, and 3.4 $229 \mathrm{~nm}$ gold nanoparticles, respectively. Though this line broadening is measurable and indicates an 230 increasingly heterogeneous surface with smaller nanoparticle sizes, the broadening is not sufficiently 231 large as to mask significant (e.g. $0.4 \mathrm{eV}$ ) shifts in the core-level electronic structure of the Au 232 nanoparticles. Therefore, our spectral analysis of the Au $4 \mathrm{f}$ core level region suggests that nanoscaling 
233 effect \#4(Table 1) does not play a significant role, as the bulk gold and the $3.4 \mathrm{~nm}, 5.3 \mathrm{~nm}$, and $9.5 \mathrm{~nm}$ in

234 diameter gold nanoparticles are all in a charge-neutral, bulk-like state.

236 The normalized raw valence region spectra for the $\mathrm{TiO}_{2}$ support and the $3.4 \mathrm{~nm}, 5.3 \mathrm{~nm}$, and $9.5 \mathrm{~nm}$ gold 237 on $\mathrm{TiO}_{2}$ are shown in figure 6.

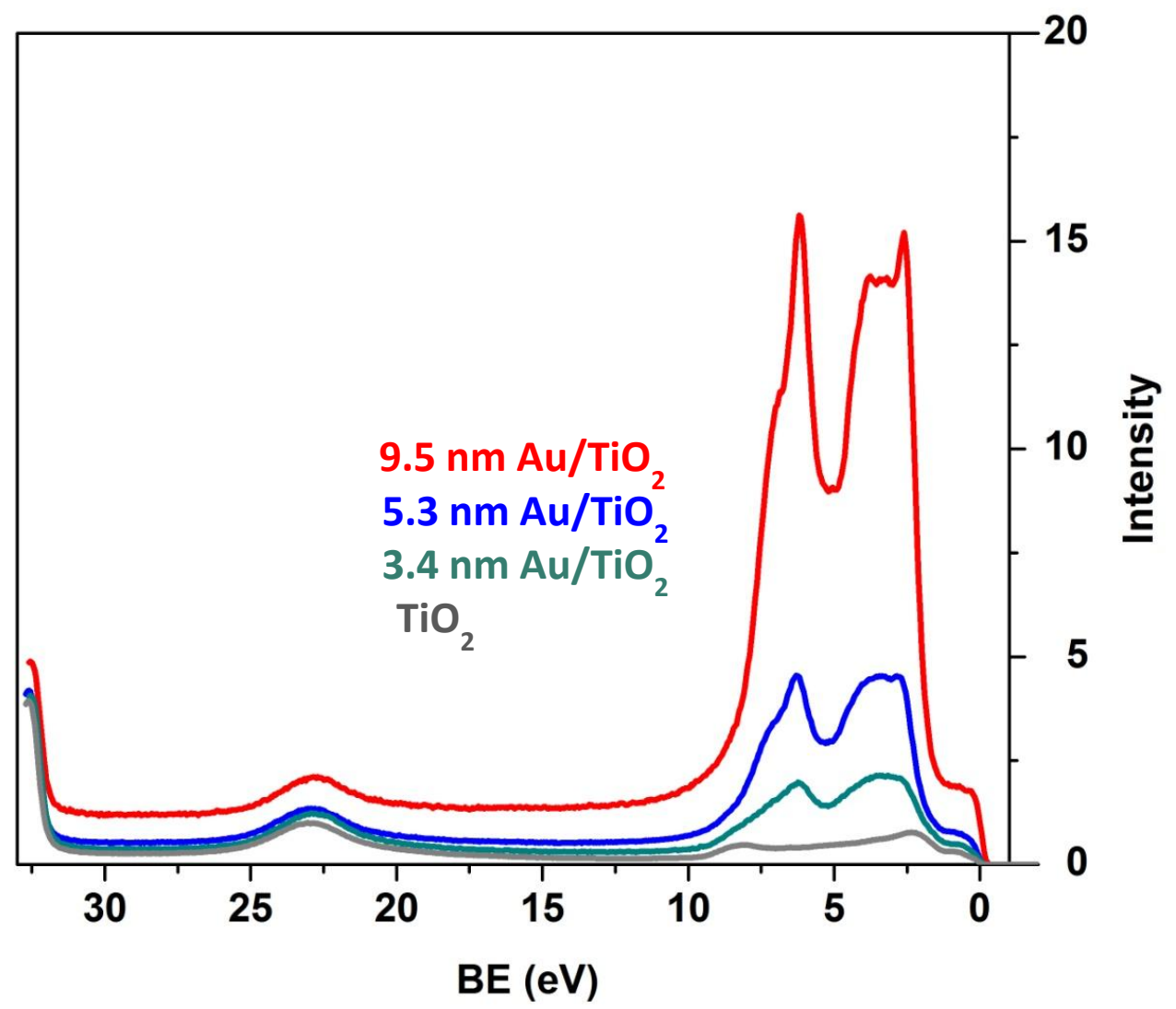

238

Figure 6. Total Valence Band X-ray Photoelectron Spectroscopy.

240 Total valence band photoelectron spectra collected using $\sim 8 \mathrm{KeV}$ hard X-rays for blank $\mathrm{TiO}_{2}$ (gray), 3.4

$241 \mathrm{~nm}$ (dark cyan), $5.3 \mathrm{~nm}$ (blue), and $9.5 \mathrm{~nm}$ (red) gold nanoparticles on $\mathrm{TiO}_{2}$ normalized with the $\mathrm{Ti} 3 \mathrm{p} \mathrm{(0)}$ 
244 As expected from figure 2, there is a very large enhancement of the gold signal in the valence region as

245 compared to the bare $\mathrm{TiO}_{2}$ support. This enhancement increases with the amount of gold deposited.

246 The valence region photoemission spectra after background $\mathrm{TiO}_{2}$ subtraction of the $3.4 \mathrm{~nm}, 5.3 \mathrm{~nm}, 9.5$

$247 \mathrm{~nm}$ diameter $\mathrm{TiO}_{2}$-supported gold nanoparticles as well as that of bulk gold are shown together in figure

2487.

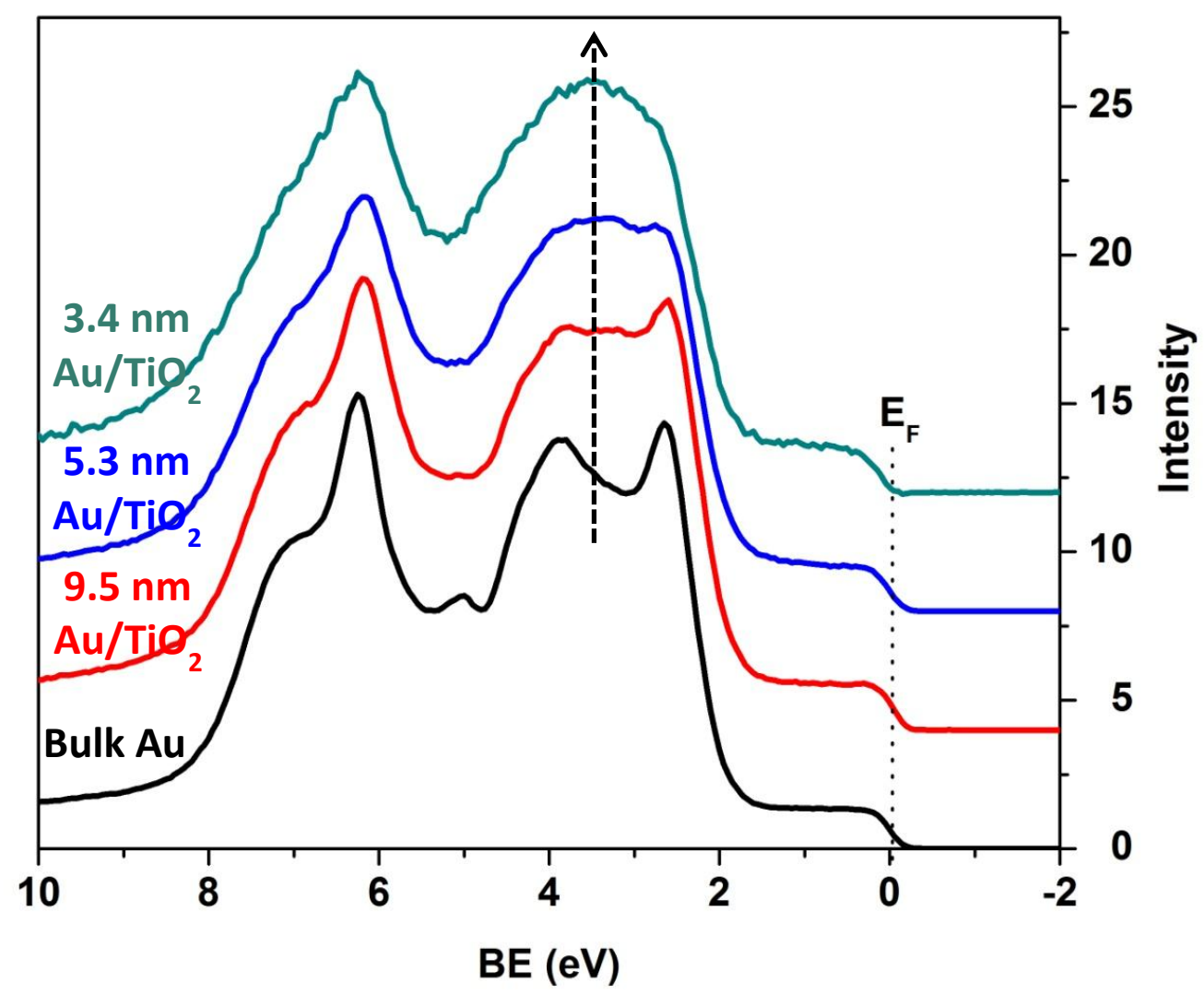

249

Figure 7. Au Valence Band.

251 Valence band spectrum of the Au d-band after $\mathrm{TiO}_{2}$ background subtraction for $3.4 \mathrm{~nm}, 5.3 \mathrm{~nm}$, and 9.5 
254 The scheme used to reference the binding energy scale and procedures relating to the normalization 255 and subtraction for all samples are described in the Supporting Information.

The main features of the gold valence band are evident in all four spectra. There is a broad and 257 flat band related to $d$ states re-hybridized with $s / p$ states [49] which extends from the Fermi level 258 through the sharper and more intense part of the $\mathrm{d}$ band that spans from roughly $2 \mathrm{eV}$ to $9 \mathrm{eV}$. Although 259 there is a slight shift observed at the Fermi edge for the $3.4 \mathrm{~nm}$ Au nanoparticles, this is within the 260 estimated experimental resolution $(\sim 0.25 \mathrm{eV})$ and is within reasonable error due to the method of $\mathrm{TiO}_{2}$ 261 background subtraction (see Supporting Information). The observation of occupied states at the Fermi 262 Level implies that the gold is metallic, therefore there is no observed quantum size effect (nanoscaling 263 effect \#5, Table 1) within this size regime $(3.4-9.5 \mathrm{~nm})$.

The sharper and more intense part of the d-band shows a number of peaks, as is expected for 265 the bulk gold valence band spectrum $[50,51]$. To facilitate comparisons among all samples, we will 266 focus our attention on features observed in the vicinity of $3.5 \mathrm{eV}$. For bulk gold, this feature consists of a 267 trough surrounded by two clear peaks. The data shows a clear trend with particle size regarding this 268 feature; the trough gradually fills in as the gold nanoparticle size decreases, until, at the smallest size $269(3.4 \mathrm{~nm})$, there is only one broad peak around 3.5 eV. While there are other, more subtle changes in the 270 valence band spectra, this particular difference among the samples is the most prominent. Morphology and support can play significant roles in the electronic structure of $\mathrm{Au}$ 272 nanoparticles. Previously, it was found for thin Au nanoparticles several monolayers thick on $\mathrm{Al}_{2} \mathrm{O}_{3}[29]$ 273 and $\mathrm{NiO}[16]$ and $\mathrm{Au}$ mono/bi/multi-layers on $\mathrm{TiO}_{x}[19]$ that the lower binding energy peak of the $\mathrm{Au} \mathrm{d}$ 274 band shifts to higher binding energies. We do not observe this shift, which suggests that such a shift may 275 be related to an intimate contact with the support since our nanoparticles are spherical and are not 276 intimately contacting the $\mathrm{TiO}_{2}$. In support of this hypothesis is other work on spherical gold 
nanoparticles $1.6-10 \mathrm{~nm}$ in diameter on a "non-interacting" carbon support, which found that the two

278 main $d$ band features remain essentially the same [30]. We thus attribute the filling in of the trough in

279 the d states observed at $\sim 3.5 \mathrm{eV}$ to an intrinsic gold nanoparticle effect, not a support effect, which synchrotron radiation enables us to observe more clearly within the valence band region than observed by others using more conventional methods.

\subsubsection{Valence Band Hypothesis, Nanoscaling effects \#1a and/or \#2 (Table 1)?}

It has been suggested previously that undercoordinated Au atoms at the nanoparticle surface

with coordination number $(\mathrm{CN}) \quad 6 \& 7$ are the most active sites for the CO oxidation reaction [10].

Undercoordinated sites at the surface can facilitate the reaction's rate determining step and activate energy d-states, which leads to stronger adsorbate interactions [10]. As the nanoparticle size decreases, the ratio of surface atoms and especially undercoordinated surface atoms to bulk atoms increases, which could, at least in part, explain the observed filling in of the d-band of Au with decrease in size.

Straining lattice parameter is also expected to have an effect on both its catalytic activity as well as its $d$ band structure [53], and therefore could, at least in part, explain the changes we observe in the $d$ band of our supported Au nanoparticles. In light of this, we attempted to address lattice-straining effects by measuring the $d$ spacing of a variety of differently sized Au nanoparticles in HRTEM. The variability in the derived Au-Au bond length using this method, in some cases greater than $3 \%$, precludes us from making any conclusive statements regarding potential straining of the lattice. Nevertheless, previous EXAFS studies have shown that the overall compressive strain in Au nanoparticles above $\sim 3 \mathrm{~nm}$ 
strain in the nanoparticle should not be a major factor. Surface and near surface straining, however,

301 plays a critical role in gold nanoparticles 3-5 nm in diameter, as determined by diffraction experiments

302 [57] and therefore must be considered as a possible cause of the observed changes in electronic 303 structure.

Therefore, our spectroscopic measurements of the Au valence band (figure 7) suggest that the

305 filling in of the trough in the $d$ states with decreasing nanoparticle size can be attributed to an increase

306 in the proportion of surface gold atoms probed by the XPS experiment that contain an increasing

307 proportion of undercoordinated Au atoms (eg. CN 6 and 7) and/or that exhibit surface (near surface)

308 strain.

Our approach to test the coordination number and/or surface straining hypothesis is to 310 computationally generate the gold d band for each of the $3.4 \mathrm{~nm}, 5.3 \mathrm{~nm}, 9.5 \mathrm{~nm}$ nanoparticles and bulk 311 gold considering the effects of both coordination number and strain and then compare with the 312 experimental XPS results. To realize this, we consider that each gold atom within the truncated 313 octahedron model (figure 4) has its d band fully specified by $\mathrm{CN}$ and either no strain or a reasonable 314 degree of contractile strain (3\%). For these calculations, we use periodic DFT and obtain d band spectra 315 of gold atom coordination numbers of $6,7,8,9$ and 12 using model periodic surfaces of $\mathrm{Au}(111)$, $316 \mathrm{Au}(100), A u(110), A u(211)$, and $A u(421)$ slabs (see supporting information) that are either unstrained or 317 contractile strained in all $X-Y-Z$ by $3 \%$. 320 strained. 


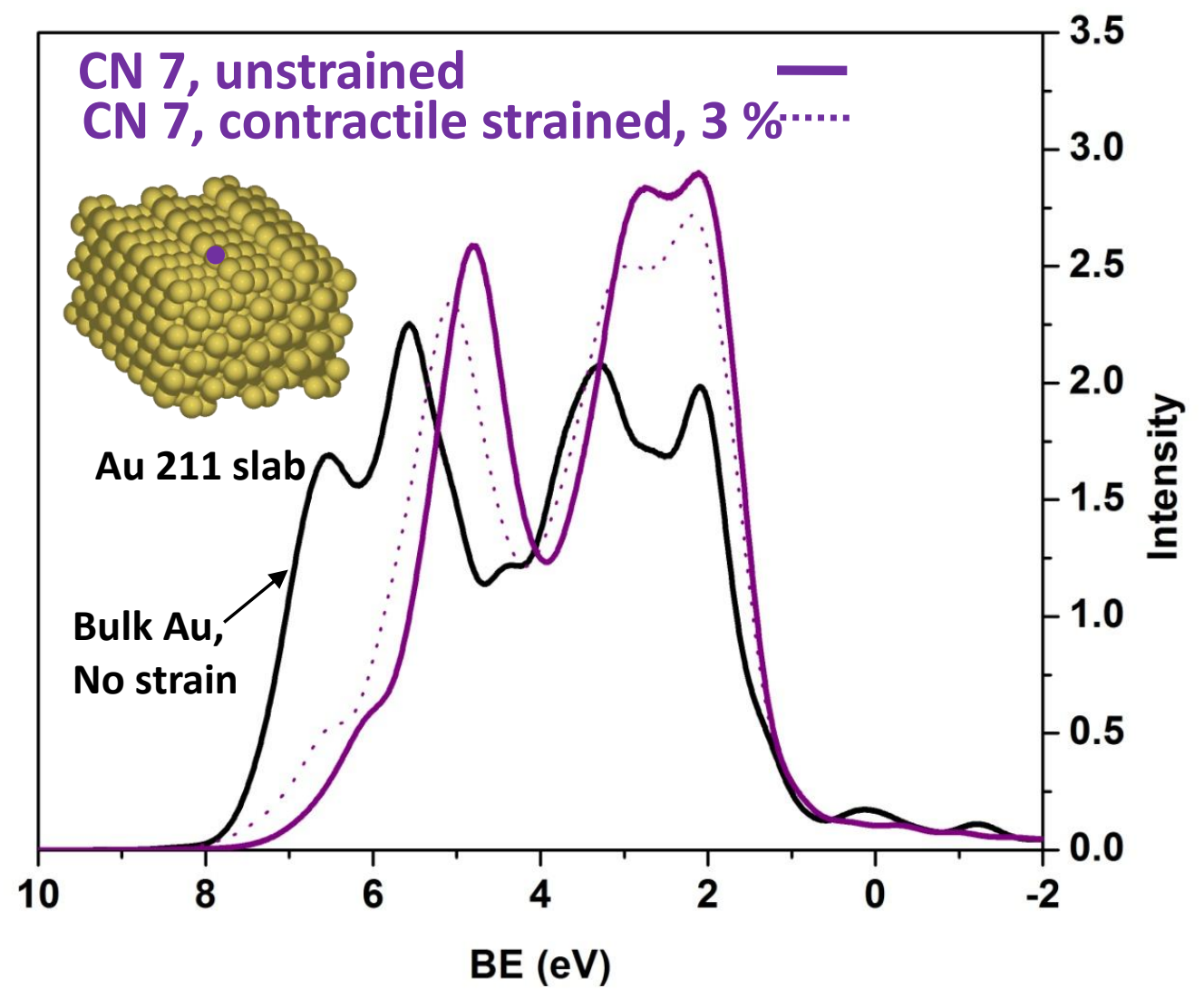

322

323

324

325

326

327 band measurements of supported Au nanoparticles, we observe the filling in of the trough. This lends

Figure 8. Calculated Au Step Edge Atom D-band.

The calculated $d$ band of the step edge atoms (CN 7) with or without strain is significantly different from that of bulk, unstrained atoms (CN 12). 3 \% contractile strain on CN 7 atoms resulted in a slight broadening. Inset: $\mathrm{An} \mathrm{Au}(211)$ slab with CN 7 step edge atom.

The $d$ band for gold atoms with $\mathrm{CN} 7$ is markedly shifted toward the Fermi level compared to the $d$ band of CN 12 atoms, with some broadening of the d-band due to the effects of strain. Interestingly, the d band of $\mathrm{CN} 7$ atoms (strained or unstrained) peaks at approximately the same region where, in our d credence to the hypotheses that the surface coordination number and/or surface (or near surface) 
333 straining that can explain the systematic changes in the $\mathrm{d}$ band measurements at approximately $3.5 \mathrm{eV}$ 334 (figure 7).

The d-DOS of each coordination number Au atom (strained or unstrained) is then inputted into 336 the truncated octahedron geometric model (or a simple flat surface model for the bulk Au), taking into 337 account effects such as XPS attenuation through the Au atoms, finite nanoparticle size distribution, and 338 the expected geometrical statistics of Au atoms with various coordination number within the truncated 339 octahedron model of each relevant sizes. In total, this model appropriately captures some of the most 340 important experimental features that are expected to be present within the measured spectra so that a 341 one to one comparison can be made between the measured and the calculated spectra. The full model 342 is described in detail in the supporting information.

Figure $9 \mathrm{~A}-\mathrm{C}$ shows the calculated d-DOS for the three nanoparticle sizes and bulk Au for the 344 cases where all Au atoms are strained ( $3 \%$ contraction) (figure 9A), only the top layer Au atoms are 345 strained ( $3 \%$ contraction) (figure 9B), and where none of the Au atoms are strained (figure 9C), and 346 compares these cases to the measurement (figure 9D). 


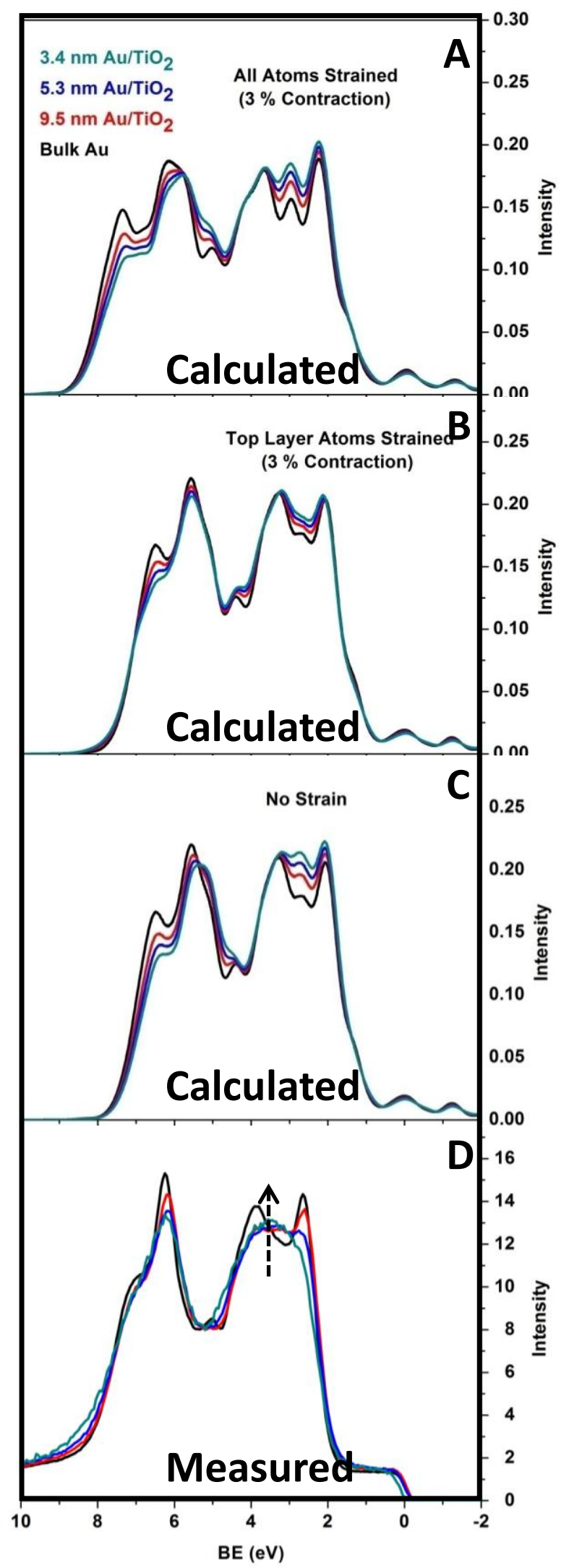


The top three graphs show the effects of $3 \%$ contractile strain strain on all atoms (A), top layer atoms

352 The important observation from this comparison is that there is a filling in effect in the $\sim 3.5 \mathrm{eV}$ region

353 for all calculated d-bands (figure 9A-C) with a decrease in nanoparticle size. This effect is present 354 regardless of whether all atoms are strained, only top layer atoms are strained, or no atoms are 355 strained, which strongly suggests that the observed filling in of the d-band trough around $3.5 \mathrm{eV}$ in the 356 measured spectra (figure 9D) is primarily due to coordination number effects only and less so to surface 357 (or near surface) strain. While surface (or near surface) strain is still likely present and could potentially 358 still have an impact on the catalytic activity, it apparently has little effect on the d-band structure of Au 359 within our system.

The other differences that are observed in regions outside of where the $d$-band trough is present ( $\sim 3.5 \mathrm{eV})$ should be mentioned briefly, keeping in mind their peripheral nature to our main 362 conclusion. 1. A small shift in relative binding energies of the $d$-band structure comparing Figures $9 \mathrm{~A}-\mathrm{C}$ to Figure 9D, which indicates that the theoretical method is appropriately capturing the d-band of $A u$, 364 but is not perfect, given well-known relativistic effects in Au [34]. 2. An obvious broadening of the entire $365 d$-band upon compressing the entire lattice by 3\% (Figure 3A vs. Figure 3C), which is expected for 366 transition metals with $d$ electron counts 5-10 [53]. 3. The discrepancy in the trend in the d-band 367 intensities at $>\sim 5.5 \mathrm{eV}$ compared between any of the calculated and the measured cases, which does 368 not yet have an explanation. 4. There is a discrepancy in the intensities in the $0-2 \mathrm{eV}$ region between 369 any of the calculated and measured $d$-bands. This region is well known to exhibit the low binding 370 energy component of the relatively flat $s p-d$ re-hybridization states in the measured spectra $[30,49]$ and 371 may be difficult to capture by our methods of calculation. 
373 decrease in size is primarily due to an increased percentage of surface Au atoms, an increasing 374 proportion of which are undercoordinated (CN 6 and 7).

376 function of size should be present regardless of the support material, however different support

377 materials could still potentially influence the Au electronic structure through other means, e.g. if the 378 support directs the shape of the gold nanoparticle to expose more or fewer of the low CN gold sites.

379 These low CN sites (steps [CN 7] and corners [CN6]) are thought by some to be the active sites in gas 380 phase $\mathrm{CO}$ oxidation catalysis $[8,58]$. Therefore, the filling in of the trough in the gold $d$ band with 381 decreasing nanoparticle size can be attributed to nanoscaling effect \# 1a (Table 1), which helps explain 382 the known catalytic activity of $\mathrm{Au}$ at the nano-scale. This effect is categorized as a geometric effect where the local geometric structure of the atoms ultimately dictates electronic structure.

\subsubsection{Valence Band Hypothesis, considering other effects}

385 While the $\mathrm{CN}$ of atoms at the gold surface can at least partially explain the experimentally observed 386 filling in of the $d$ band trough, we have also considered a number of alternative possibilities: gold at the 387 interface with $\mathrm{TiO}_{2}$, including unique perimeter sites; amorphization; gold atoms at the surface to which 388 molecules $\left(\mathrm{C}, \mathrm{N}_{2}, \mathrm{O}_{2}\right)$ are adsorbed; and re-hybridization of the d states with s or $p$ states with size. nanoparticles yielded a large fraction of such sites; this, however, is not the case for the nanoparticles studied herein as they possess a spherical geometry with less than $1 \%$ of the atoms representing 
Therefore, perimeter and interface sites can be ruled out as the cause for the experimentally observed

396 filling in of the $d$ band trough. We cannot, however, rule out the possibility that perimeter sites are the 397 active sites for catalysis (nanoscaling effect \#3, Table 1); such a determination is outside the scope of the present manuscript.

We have assumed that the small gold nanoparticles can be modeled as crystalline truncated octahedron or other suitable crystalline polyhedra, however, at these small nanoparticle sizes, 401 amorphization and disorder could play a very large role. Indeed, it was found that gold nanoparticles of $4021.6 \mathrm{~nm}$ in diameter exhibit substantial atomic disorder [59] when immersed in solvent. However, when 403 studied in the dry state, without solvent, much of the disordering is absent. Therefore, given that the 404 nanoparticles in our study are much larger than those in the aforementioned literature reference and 405 that we are studying "dry" nanoparticles, our nanoparticles should resemble more a crystalline state 406 than a disordered, glass-like state. The TEM images shown in figure 3 are consistent with this view. how they might fill in of the trough in the $d$ band; we have ruled out this possibility as well. Most of the common adsorbates, even for $\mathrm{Au}(110)$ surfaces (CN 7), desorb from the gold surface considerably below room temperature [52] and especially at low pressure. The only gold surface sites that might be 411 considered as reactive to these adsorbates are corner sites ( $C N$ 6), which comprise a much smaller 412 proportion of the total gold atoms within the gold nanoparticles than the magnitude of the proportional 413 change observed within the $d$ band (see Supporting Information). The same logic applies to the possible 414 influence of $s p-d$ re-hybridization as a function of gold nanoparticle size. The estimated perturbation of 415 the $d$ electron count due to sp- $d$ re-hybridization per Au atom over the full range of sizes measured is $4160.3 \%$ (see supporting information)[49], outside the detection capability of XPS. We can thus rule out sp- 
417 d re-hybridization playing a major role in affecting electronic structure changes as a function of gold 418 nanoparticle size within the range studied here.

We thus find that the DFT calculations for different CN Au atoms with and without 3\% compressive strain, combined with a straightforward geometric model of an Au nanoparticle, can 421 explain the observed sized dependent changes in the $d$ band. Ultimately, the presence of surface Au 422 atoms, an increasing proportion of which are undercoordinated ( $\mathrm{CN} 6$ and 7) with decreasing 423 nanoparticle size, is responsible for the filling in of the $d$ band trough we observe experimentally. As 424 undercoordinated ( $\mathrm{CN} 6$ and 7) atoms are thought to be the catalytically active sites on small supported 425 gold nanoparticles, this work reveals a link between the catalytically active sites on Au nanoparticles and 426 their electronic structure, probed by both experiment and DFT.

While the focus of this study is on nearly spherical gold nanoparticles of $3.4,5.3$, and $9.5 \mathrm{~nm}$ 428 diameter deposited onto $\mathrm{TiO}_{2}$, our approach is broadly applicable to a host of other sizes, shapes and 429 supports. The principal advantage of our approach is the use of hard X-ray, synchrotron based XPS 430 measurements that can discriminate small changes in the valence band features of gold, as portrayed 431 within the region around $3.5 \mathrm{eV}$, without major contributions from the underlying metal oxide support. 432 Measuring the valence band contributions of gold versus a $3 d / 4 d$ metal oxide support at 8 KeV versus 433 conventional Al Ka (1486 eV) XPS or standard UPS ( $40 \mathrm{eV})$ amplifies the gold $5 \mathrm{~d}$ band cross section 434 relative to the valence band contributions (metal $3 d / 4 d$ and $02 p$ ) of its support by roughly one and two 435 orders of magnitude, respectively (see supporting information). With conventional XPS or UPS, the 436 valence band signal from the $3 d / 4 d$ metal oxides would typically overwhelm that of the $5 d$ transition 437 metal. This benefit of using synchrotron-based hard x-rays can be applied toward the study of any $5 \mathrm{~d}$ 438 transition metal catalyst in which one aims to distinguish its valence band signal from $3 \mathrm{~d} / 4 \mathrm{~d}$ metals or 439 metal oxides present in the system, e.g. a $5 d$ metal supported onto a $3 d / 4 d$ metal oxide support or a 
metal alloy between $5 d$ metal and a $3 d / 4 d$ metal, as has been shown in a previous study of Pt versus $\mathrm{Cu}$

441 within a PtCu alloy catalyst [60].

\section{Conclusions}

dictate their electronic structure and how we can use the measured electronic structure to identify the

447 well-defined, thin-film $\mathrm{TiO}_{2}$ support. High-resolution TEM imaging revealed that the nanoparticles were

448 sitting nearly spherically on the $\mathrm{TiO}_{2}$ support. All three investigated sizes of Au nanoparticles as well as

449 bulk gold were found to be in a bulk-like metallic state, as determined by the Au $4 \mathrm{f}$ doublet from XPS.

450 Valence band measurements using hard x-ray XPS afforded a clearly distinguishable signals for Au 451 valence band versus that from the $\mathrm{TiO}_{2}$ support. We observe significant changes in the Au valence band 452 as a function of Au particle size. More specifically, we measured a significant increase in intensity at a 453 binding energy of $3.5 \mathrm{eV}$, a region between two valence band peaks in bulk $\mathrm{Au}$, as a function of 454 decreasing Au particle size. DFT calculations suggest that this is due to the proportional increase in 455 surface $\mathrm{Au}$ atoms, an increasing percentage of which are undercoordinated (CN 6 and 7), with a 456 decrease in gold nanoparticle size (nanoscaling effect \#1a, Table 1). This effect of coordination number 457 on d-band will likely occur irrespective of the choice of support, though different supports will likely still 458 have some impact on the electronic structure of gold, e.g. through shape effects which will influence the 459 fraction of low CN sites. By having measured discrete changes in the gold valence band structure of 460 supported gold nanoparticles using synchrotron-based hard x-rays and utilizing theoretical modeling, we 461 have elucidated how coordination number plays a crucial role in affecting the electronic properties of 462 nanoparticulate gold, which ultimately determine its catalytic activity. The approach utilized herein is of 
particular value in assessing the electronic structure of catalytically active surface sites of $5 \mathrm{~d}$ transition metal nanoparticles supported onto standard 3d/4d metal oxide supports.

\section{Acknowledgements}

This material is based upon work supported by the National Science Foundation under Grant No. 1066515. Seed funding was provided by a Hellman Faculty Scholar Award. B.N.R. thanks the National Defense Science and Engineering Graduate (NDSEG) fellowship and the Stanford Graduate Fellowship (SGF) for fellowship support. T.F.J., H.O., L.L., J.V., F.A-P, and A.N. acknowledge support from the DOE Office of Basic Energy Science to the SUNCAT Center for Interface Science and Catalysis. This work was performed in part at the Stanford Nanofabrication Facility which is supported by National Science Foundation through the NNIN under Grant ECS-9731293. The synchrotron radiation experiments were performed at the BL47XU of SPring-8 with the approval of the Japan Synchrotron Radiation Research Institute (JASRI) (Proposal No. 2009B1751). The authors thank Eiji Ikenaga for technical assistance at the Spring8 Synchrotron. B.N.R. thanks Ann Marshall for TEM preparation training. The authors would also like to acknowledge Zhebo Chen, Arnold Forman, and Jens K. Nørskov for helpful discussions.

\section{[1] M. Haruta, N. Yamada, T. Kobayashi, S. lijima, GOLD CATALYSTS PREPARED BY COPRECIPITATION FOR LOW-TEMPERATURE OXIDATION OF HYDROGEN AND OF CARBON-MONOXIDE, Journal of Catalysis, 115} (1989) 301-309.

[2] D. Andreeva, V. Idakiev, T. Tabakova, A. Andreev, R. Giovanoli, Low-temperature water-gas shift reaction on Au/alpha-Fe2O3 catalyst, Applied Catalysis a-General, 134 (1996) 275-283.

[3] A. Ueda, M. Haruta, Nitric oxide reduction with hydrogen, carbon monoxide, and hydrocarbons over gold catalysts, Gold Bulletin, 32 (1999) 3-+.

[4] T. Hayashi, K. Tanaka, M. Haruta, Selective vapor-phase epoxidation of propylene over Au/TiO2 catalysts in the presence of oxygen and hydrogen, Journal of Catalysis, 178 (1998) 566-575.

[5] G.C. Bond, P.A. Sermon, G. Webb, D.A. Buchanan, P.B. Wells, HYDROGENATION OVER SUPPORTED GOLD CATALYSTS, Journal of the Chemical Society-Chemical Communications, (1973) 444-445.

[6] J.L. Gong, Structure and Surface Chemistry of Gold-Based Model Catalysts, Chemical Reviews, 112 (2012) 2987-3054.

[7] M. Haruta, S. Tsubota, T. Kobayashi, H. Kageyama, M.J. Genet, B. Delmon, LOW-TEMPERATURE OXIDATION OF CO OVER GOLD SUPPORTED ON TIO2, ALPHA-FE2O3, AND CO3O4, Journal of Catalysis, 144 (1993) 175-192. 
[8] T.V.W. Janssens, A. Carlsson, A. Puig-Molina, B.S. Clausen, Relation between nanoscale Au particle structure and activity for CO oxidation on supported gold catalysts, Journal of Catalysis, 240 (2006) 108113. [9] A. Carlsson, A. Puig-Molina, T.V.W. Janssens, New method for analysis of nanoparticle geometry in supported fee metal catalysts with scanning transmission electron microscopy, Journal of Physical Chemistry B, 110 (2006) 5286-5293.

[10] T.V.W. Janssens, B.S. Clausen, B. Hvolbaek, H. Falsig, C.H. Christensen, T. Bligaard, J.K. Norskov, Insights into the reactivity of supported Au nanoparticles: combining theory and experiments, Topics in Catalysis, 44 (2007) 15-26.

[11] Y. Xu, M. Mavrikakis, Adsorption and dissociation of O-2 on gold surfaces: Effect of steps and strain, Journal of Physical Chemistry B, 107 (2003) 9298-9307.

[12] M. Mavrikakis, P. Stoltze, J.K. Norskov, Making gold less noble, Catalysis Letters, 64 (2000) 101-106.

[13] M.A. Brown, F. Ringleb, Y. Fujimori, M. Sterrer, H.-J. Freund, G. Preda, G. Pacchioni, Initial Formation of Positively Charged Gold on $\mathrm{MgO}(001)$ Thin Films: Identification by Experiment and Structural Assignment by Theory, Journal of Physical Chemistry C, 115 (2011) 10114-10124.

[14] E. Florez, L. Feria, F. Vines, J.A. Rodriguez, F. Illas, Effect of the Support on the Electronic Structure of Au Nanoparticles Supported on Transition Metal Carbides: Choice of the Best Substrate for $\mathrm{Au}$ Activation, Journal of Physical Chemistry C, 113 (2009) 19994-20001.

[15] N. Lopez, J.K. Norskov, T.V.W. Janssens, A. Carlsson, A. Puig-Molina, B.S. Clausen, J.D. Grunwaldt, The adhesion and shape of nanosized Au particles in a $\mathrm{Au} / \mathrm{TiO}(2)$ catalyst, Journal of Catalysis, 225 (2004) 86-94.

[16] T. Okazawa, M. Fujiwara, T. Nishimura, T. Akita, M. Kohyama, Y. Kido, Growth mode and electronic structure of Au nano-clusters on $\mathrm{NiO}(001)$ and TiO2(110), Surface Science, 600 (2006) 1331-1338.

[17] T. Risse, S. Shaikhutdinov, N. Nilius, M. Sterrer, H.-J. Freund, Gold supported on thin oxide films: From single atoms to nanoparticles, Accounts of Chemical Research, 41 (2008) 949-956.

[18] M. Zhou, A. Zhang, Z. Dai, Y.P. Feng, C. Zhang, Strain-Enhanced Stabilization and Catalytic Activity of Metal Nanoclusters on Graphene, Journal of Physical Chemistry C, 114 (2010) 16541-16546.

[19] M. Chen, Y. Cai, Z. Yan, D.W. Goodman, On the origin of the unique properties of supported Au nanoparticles, Journal of the American Chemical Society, 128 (2006) 6341-6346.

[20] D.C. Meier, D.W. Goodman, The influence of metal cluster size on adsorption energies: CO adsorbed on Au clusters supported on TiO2, Journal of the American Chemical Society, 126 (2004) 1892-1899.

[21] K. Okazaki, S. Ichikawa, Y. Maeda, M. Haruta, M. Kohyama, Electronic structures of Au supported on TiO2, Applied Catalysis a-General, 291 (2005) 45-54.

[22] M. Valden, X. Lai, D.W. Goodman, Onset of catalytic activity of gold clusters on titania with the appearance of nonmetallic properties, Science, 281 (1998) 1647-1650.

[23] C.H. Christensen, J.K. Norskov, A molecular view of heterogeneous catalysis, The Journal of chemical physics, 128 (2008) 182503.

[24] T. Jiang, D.J. Mowbray, S. Dobrin, H. Falsig, B. Hvolbaek, T. Bligaard, J.K. Norskov, Trends in CO Oxidation Rates for Metal Nanoparticles and Close-Packed, Stepped, and Kinked Surfaces, Journal of Physical Chemistry C, 113 (2009) 10548-10553.

[25] J.K. Norskov, T. Bligaard, B. Hvolbaek, F. Abild-Pedersen, I. Chorkendorff, C.H. Christensen, The nature of the active site in heterogeneous metal catalysis, Chemical Society Reviews, 37 (2008) 21632171. catalysts, Nature Chemistry, 1 (2009) 37-46.

[27] V.R. Stamenkovic, B.S. Mun, M. Arenz, K.J.J. Mayrhofer, C.A. Lucas, G. Wang, P.N. Ross, N.M. Markovic, Trends in electrocatalysis on extended and nanoscale Pt-bimetallic alloy surfaces, Nature 541 Materials, 6 (2007) 241-247. 
542

543

544

545

546

547

548

549

550

551

552

553

554

555

556

557

558

559

560

561

562

563

564

565

566

567

568

569

570

571

572

573

574

575

576

577

578

579

580

581

582

583

584

585

586

587

[28] T. Okazawa, M. Kohyama, Y. Kido, Electronic properties of Au nano-particles supported on stoichiometric and reduced TiO2 (110) substrates, Surface Science, 600 (2006) 4430-4437.

[29] M.-F. Luo, C.-C. Wang, G.-R. Hu, W.-R. Lin, C.-Y. Ho, Y.-C. Lin, Y.-J. Hsu, Active Alloying of Au with Pt in Nanoclusters Supported on a Thin Film of $\mathrm{Al}(2) \mathrm{O}(3) / \mathrm{NiAl}(100)$, Journal of Physical Chemistry C, 113 (2009) 21054-21062.

[30] H.G. Boyen, T. Herzog, G. Kastle, F. Weigl, P. Ziemann, J.P. Spatz, M. Moller, R. Wahrenberg, M.G. Garnier, P. Oelhafen, X-ray photoelectron spectroscopy study on gold nanoparticles supported on diamond, Physical Review B, 65 (2002).

[31] H.G. Boyen, G. Kastle, F. Weigl, P. Ziemann, G. Schmid, M.G. Garnier, P. Oelhafen, Chemically induced metal-to-insulator transition in Au55 clusters: effect of stabilizing ligands on the electronic properties of nanoparticles, Physical review letters, 87 (2001) 276401.

[32] M. Buttner, P. Oelhafen, XPS study on the evaporation of gold submonolayers on carbon surfaces, Surface Science, 600 (2006) 1170-1177.

[33] G.K. Wertheim, S.B. Dicenzo, S.E. Youngquist, UNIT CHARGE ON SUPPORTED GOLD CLUSTERS IN PHOTOEMISSION FINAL-STATE, Physical Review Letters, 51 (1983) 2310-2313.

[34] G.C. Bond, D.T. Thompson, Catalysis by gold, Catalysis Reviews-Science and Engineering, 41 (1999) 319-388.

[35] J.J. Yeh, I. Lindau, ATOMIC SUBSHELL PHOTOIONIZATION CROSS-SECTIONS AND ASYMMETRY PARAMETERS - 1 LESS-THAN-OR-EQUAL-TO Z LESS-THAN-OR-EQUAL-TO 103, Atomic Data and Nuclear Data Tables, 32 (1985) 1-155.

[36] P. Giannozzi, S. Baroni, N. Bonini, M. Calandra, R. Car, C. Cavazzoni, D. Ceresoli, G.L. Chiarotti, M. Cococcioni, I. Dabo, A. Dal Corso, S. de Gironcoli, S. Fabris, G. Fratesi, R. Gebauer, U. Gerstmann, C. Gougoussis, A. Kokalj, M. Lazzeri, L. Martin-Samos, N. Marzari, F. Mauri, R. Mazzarello, S. Paolini, A. Pasquarello, L. Paulatto, C. Sbraccia, S. Scandolo, G. Sclauzero, A.P. Seitsonen, A. Smogunov, P. Umari, R.M. Wentzcovitch, QUANTUM ESPRESSO: a modular and open-source software project for quantum simulations of materials, Journal of Physics-Condensed Matter, 21 (2009).

[37] A. Dal Corso, A.M. Conte, Spin-orbit coupling with ultrasoft pseudopotentials: Application to Au and Pt, Physical Review B, 71 (2005).

[38] D. Vanderbilt, SOFT SELF-CONSISTENT PSEUDOPOTENTIALS IN A GENERALIZED EIGENVALUE FORMALISM, Physical Review B, 41 (1990) 7892-7895.

[39] Vanharde.R, F. Hartog, STATISTICS OF SURFACE ATOMS AND SURFACE SITES ON METAL CRYSTALS, Surface Science, 15 (1969) 189-\&.

[40] B.R. Cuenya, S.H. Baeck, T.F. Jaramillo, E.W. McFarland, Size- and support-dependent electronic and catalytic properties of Au-0/Au3 + nanoparticles synthesized from block copolymer micelles, Journal of the American Chemical Society, 125 (2003) 12928-12934.

[41] M.G. Mason, ELECTRONIC-STRUCTURE OF SUPPORTED SMALL METAL-CLUSTERS, Physical Review B, 27 (1983) 748-762.

[42] Y. Sohn, D. Pradhan, A. Radi, K.T. Leung, Interfacial Electronic Structure of Gold Nanoparticles on Si(100): Alloying versus Quantum Size Effects, Langmuir, 25 (2009) 9557-9563.

[43] Z.X. Yang, R.Q. Wu, Origin of positive core-level shifts in Au clusters on oxides, Physical Review B, 67 (2003).

[44] P.H. Citrin, G.K. Wertheim, Y. Baer, CORE-LEVEL BINDING-ENERGY AND DENSITY OF STATES FROM SURFACE ATOMS OF GOLD, Physical Review Letters, 41 (1978) 1425-1428.

[45] J. Radnik, C. Mohr, P. Claus, On the origin of binding energy shifts of core levels of supported gold nanoparticles and dependence of pretreatment and material synthesis, Physical Chemistry Chemical Physics, 5 (2003). 
[46] M.P. Seah, G.C. Smith, M.T. Anthony, AES - ENERGY CALIBRATION OF ELECTRON SPECTROMETERS .1. AN ABSOLUTE, TRACEABLE ENERGY CALIBRATION AND THE PROVISION OF ATOMIC REFERENCE LINE ENERGIES, Surface and Interface Analysis, 15 (1990) 293-308.

[47] N. Weiher, E. Bus, L. Delannoy, C. Louis, D.E. Ramaker, J.T. Miller, J.A. van Bokhoven, Structure and oxidation state of gold on different supports under various $\mathrm{CO}$ oxidation conditions, Journal of Catalysis, 240 (2006) 100-107.

[48] H.G. Boyen, A. Ethirajan, G. Kastle, F. Weigl, P. Ziemann, G. Schmid, M.G. Garnier, M. Buttner, P. Oelhafen, Alloy formation of supported gold nanoparticles at their transition from clusters to solids: Does size matter?, Physical Review Letters, 94 (2005).

[49] J.A. van Bokhoven, J.T. Miller, d electron density and reactivity of the $d$ band as a function of particle size in supported gold catalysts, Journal of Physical Chemistry C, 111 (2007) 9245-9249. [50] U. Gelius, L. Asplund, E. Basilier, S. Hedman, K. Helenelund, K. Siegbahn, A HIGH-RESOLUTION MULTIPURPOSE ESCA INSTRUMENT WITH X-RAY MONOCHROMATOR, Nuclear Instruments \& Methods in Physics Research Section B-Beam Interactions with Materials and Atoms, 229 (1984) 85-117. [51] Z. Hussain, N.F.T. Hall, L.F. Wagner, S.P. Kowalczyk, C.S. Fadley, K.A. Thompson, R.L. Dod, BRILLOUIN-ZONE AVERAGING IN ANGLE-RESOLVED X-RAY PHOTOEMISSION FROM VALENCE LEVELS OF SINGLE-CRYSTAL GOLD AND ALUMINUM, Solid State Communications, 25 (1978) 907-911.

[52] R. Meyer, C. Lemire, S.K. Shaikhutdinov, H. Freund, Surface chemistry of catalysis by gold, Gold Bulletin, 37 (2004) 72-+.

[53] P. Strasser, S. Koh, T. Anniyev, J. Greeley, K. More, C.F. Yu, Z.C. Liu, S. Kaya, D. Nordlund, H. Ogasawara, M.F. Toney, A. Nilsson, Lattice-strain control of the activity in dealloyed core-shell fuel cell catalysts, Nature Chemistry, 2 (2010) 454-460.

[54] A. Balerna, E. Bernieri, P. Picozzi, A. Reale, S. Santucci, E. Burattini, S. Mobilio, A STRUCTURAL INVESTIGATION ON SMALL GOLD CLUSTERS BY EXAFS, Surface Science, 156 (1985) 206-213.

[55] A. Balerna, E. Bernieri, P. Picozzi, A. Reale, S. Santucci, E. Burattini, S. Mobilio, EXTENDED X-RAYABSORPTION FINE-STRUCTURE AND NEAR-EDGE-STRUCTURE STUDIES ON EVAPORATED SMALL CLUSTERS OF AU, Physical Review B, 31 (1985) 5058-5065.

[56] J.T. Miller, A.J. Kropf, Y. Zha, J.R. Regalbuto, L. Delannoy, C. Louis, E. Bus, J.A. van Bokhoven, The effect of gold particle size on Au-Au bond length and reactivity toward oxygen in supported catalysts, Journal of Catalysis, 240 (2006) 222-234.

[57] W.J. Huang, R. Sun, J. Tao, L.D. Menard, R.G. Nuzzo, J.M. Zuo, Coordination-dependent surface atomic contraction in nanocrystals revealed by coherent diffraction, Nature Materials, 7 (2008) 308-313. [58] H. Falsig, B. Hvolbaek, I.S. Kristensen, T. Jiang, T. Bligaard, C.H. Christensen, J.K. Norskov, Trends in the catalytic $\mathrm{CO}$ oxidation activity of nanoparticles, Angewandte Chemie-International Edition, 47 (2008) 4835-4839.

[59] V. Petkov, N. Bedford, M.R. Knecht, M.G. Weir, R.M. Crooks, W. Tang, G. Henkelman, A. Frenkel, Periodicity and atomic ordering in nanosized particles of crystals, Journal of Physical Chemistry C, 112 (2008) 8907-8911.

[60] T. Anniyev, H. Ogasawara, M.P. Ljungberg, K.T. Wikfeldt, J.B. MacNaughton, L.A. Naslund, U. Bergmann, S. Koh, P. Strasser, L.G.M. Pettersson, A. Nilsson, Complementarity between high-energy photoelectron and L-edge spectroscopy for probing the electronic structure of $5 \mathrm{~d}$ transition metal catalysts, Physical Chemistry Chemical Physics, 12 (2010) 5694-5700. 


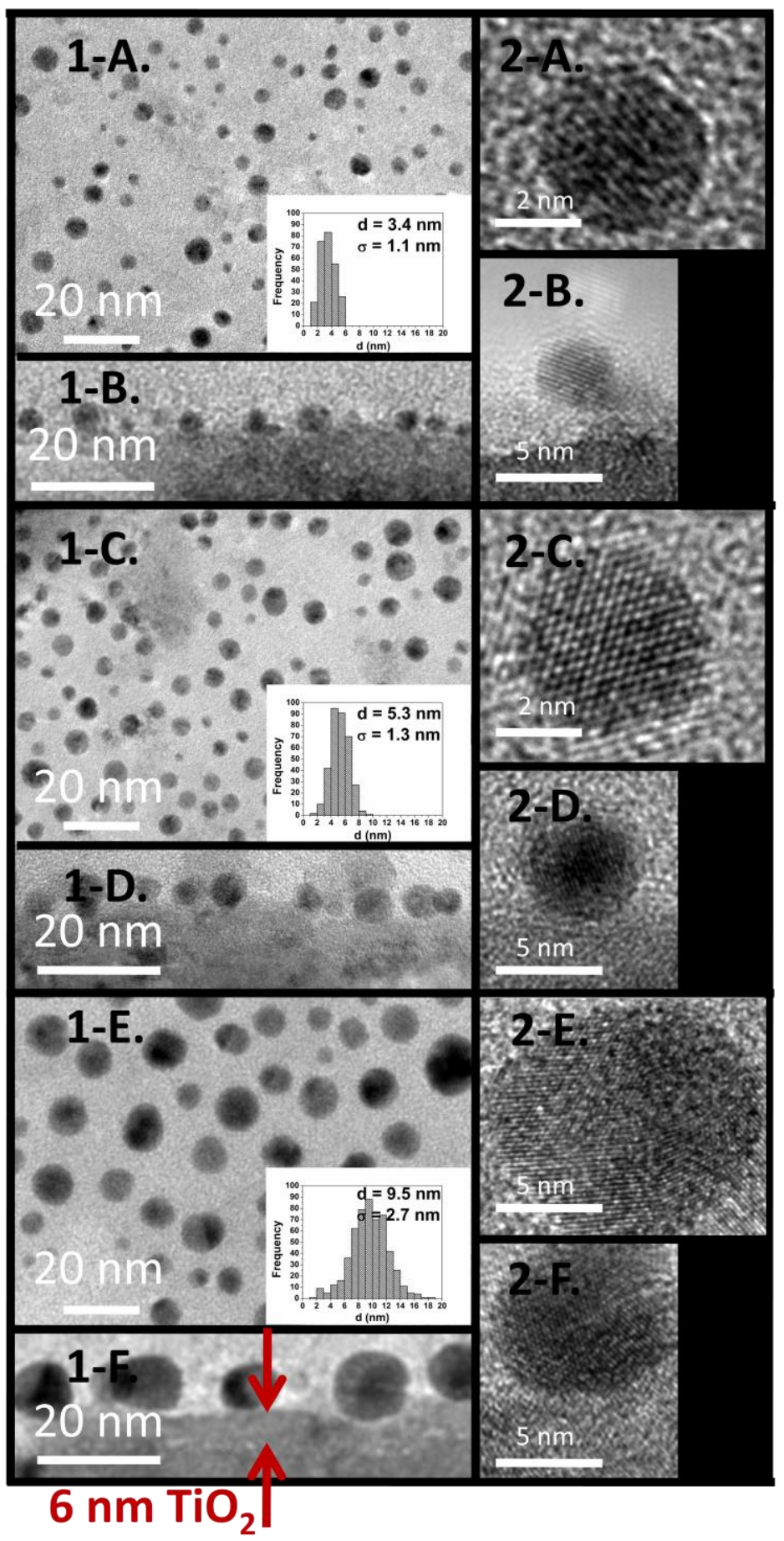

Figure 3. Au Nanoparticle Imaging.

Morphology, size, size distribution, and atomic scale features of gold nanoparticles on $\mathrm{TiO}_{2}$ as observed by TEM. The gold nanoparticles of $3 \AA, 8 \AA$, and $20 \AA$ nominal deposition thickness are shown in the top row, middle row and bottom row, respectively. The TEM low resolution and high resolution images of the gold nanoparticles are shown in 1-A through 1-F and 2-A through 2-F, respectively, with the top- 
down (plan) views noted $A, C$, and $E$ and with the cross-sectional views noted $B, D$, and $F$. Additionally, the histograms for the TEM plan -view based nanoparticle diameter distributions are shown as insets to 1-A, 1-C, and 1-E. Finally, a $\mathrm{TiO}_{2}$ layer thickness of around $6 \mathrm{~nm}$ is indicated on 1-F with arrows that define the boundaries of the oxide layer above the titanium metal. 


\section{${ }^{\star}$ Graphical Abstract (for review)}

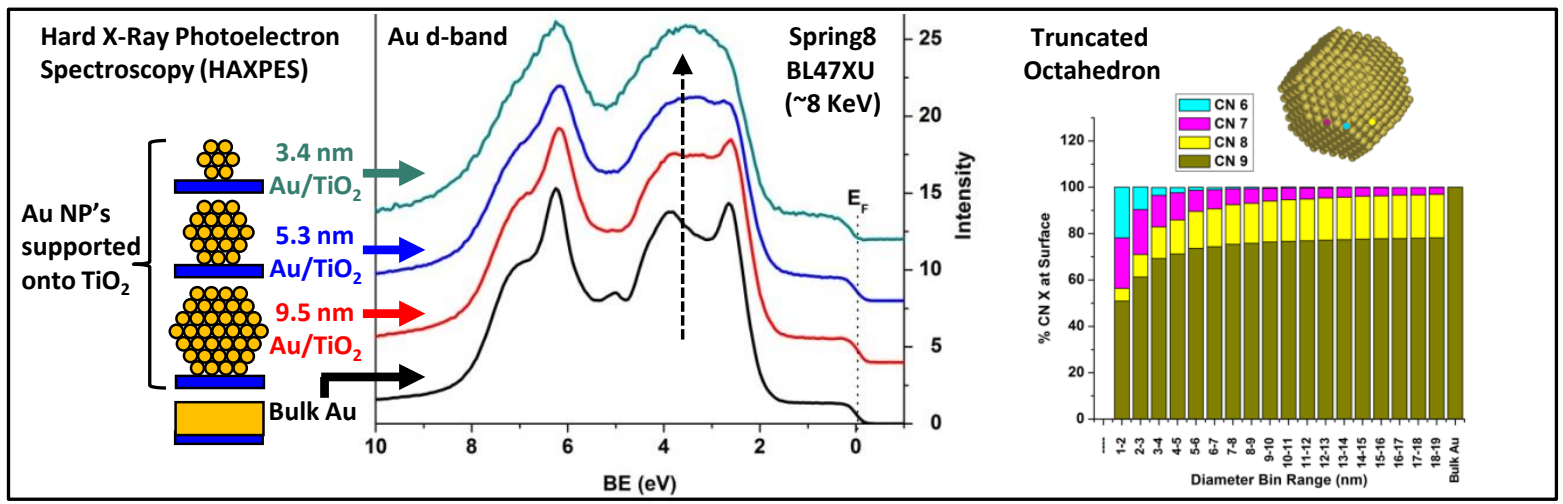

